\section{Increased BMI and the risk of incident disability in elderly people}

Several studies have shown that the association of increased BMI with increased mortality is reversed among the elderly population, in whom optimal life expectancy is associated with being overweight or obese. Al Snih et al. studied the association between BMI and mortality, and, furthermore, the relationship between BMI and incident disability - an important aspect of health and wellbeing in elderly people.

The data used in this study originated from the EPESE (Established Populations for Epidemiologic Studies of the Elderly) studies. The 12,725 participants included in the final analysis were aged $\geq 65$ years, and were not functionally disabled at baseline. Disability, which was selfreported, was defined as requiring help to perform $\geq 1$ of 7 activities of daily living, such as bathing, walking across a small room, or using the toilet. Participants were followed up for 7 years.

The BMI score associated with the lowest risk of disability was significantly lower than that associated with the lowest risk of mortality, with or without adjustment for all baseline comorbidities (hazard ratio 24.2 versus 27.3 and 24.1 versus 26.1, respectively; $P<0.001$ for both comparisons). If smokers and participants who died within the first 2 years of follow-up were excluded, no significant difference was observed.

The authors conclude that elevated BMI in people aged over 65 years is associated with an increased risk of experiencing disability. Optimum BMI scores in this population should, therefore, be determined with due consideration of this risk, in addition to the risk of mortality.

Original article Al Snih S et al. (2007) The effect of obesity on disability vs mortality in older Americans. Arch Intern Med 167: 774-780

\section{Thyroid hormone use does not lead to increased mortality in older women}

Despite the widespread use of thyroid hormone in older women (aged $\geq 65$ years), its effect on mortality has not been well studied. To investigate the relationship between thyroid hormone usage, thyroid dysfunction and mortality in older women, Bauer et al. performed a prospective cohort study in patients with and without thyroid disorders.
Of the 9,449 women evaluated, $12 \%$ were taking thyroid hormone at baseline, with a mean treatment duration of 15.8 years. A history of hyperthyroidism was reported in $9.4 \%$ of patients. Over 11.9 years' follow-up, 3,159 deaths were recorded, mainly from cardiovascular causes and cancer.

Overall, age-adjusted analyses showed that a history of hyperthyroidism was associated with increased mortality (relative hazard [RH] $1.20,95 \% \mathrm{Cl} 1.06-1.36$, after adjusting for thyroid hormone use, weight, health, exercise levels, diabetes, hypertension, and use of alcohol, tobacco, estrogen and thiazides). A history of hyperthyroidism was also associated with increased cardiovascular mortality on multivariate analysis $(\mathrm{RH} 1.46,95 \% \mathrm{Cl} 1.20-1.77)$. This association was similar among the subset of women in whom TSH was measured.

Although age-adjusted analyses showed an association between thyroid hormone use and mortality, no association was seen on multivariate analysis. Likewise, no significant association was observed between thyroid hormone use and cardiovascular or cancer mortality, nor between the level of TSH and mortality in a subcohort of 487 patients.

While the results of this study are reassuring for elderly women taking thyroid hormone, further confirmatory studies are needed.

Original article Bauer DC et al. (2007) Thyroid hormone use, hyperthyroidism and mortality in older women. Am J Med 120: 343-349

\section{Sonoelastography can diagnose cervical lymph-node metastasis with high accuracy}

Sonoelastography, an ultrasound imaging technique that measures the elasticity of soft tissues under gentle compression, could be a promising modality for the diagnosis of cervical lymph-node metastases. Lyshchik et al. evaluated the technique in a prospective study that included 43 patients with suspected thyroid or hypopharyngeal cancer who underwent ultrasound evaluation before surgery. A total of 141 lymph nodes were examined. Sonoelastography was found to be $92 \%$ accurate in differentiating benign from metastatic cervical lymph nodes.

Patients underwent gray-scale and power Doppler ultrasonography to measure the 